\title{
Una Meditación de la Ciencia
}

\author{
Dr. Raúl Fornet-Betancourt / Universidad de \\ Bremen. ${ }^{1}$
}

Trabajo recepcionado: mayo 2007

Trabajo aceptado: junio 2007

\section{RESUMEN}

Haciendo una crítica intercultural a la ciencia moderna, el autor del artículo propone pensar la ciencia como un pensar meditativo que recobra el sentido y que se percata del significado de su tarea ante la ciencia, por ello toma conciencia de que debe ir más allá de la crítica. Para esta crítica no basta con censurar o poner de manifiesto las desviaciones de la ciencia en sus aplicaciones, sino que hay que sobrepasar este horizonte, poniendo a la ciencia en su lugar. Saber, sin embargo, qué lugar le corresponde a la ciencia, implica saber qué sentido tienen el conocimiento humano y la actividad cognitiva del ser humano en general dentro del desarrollo de la vida, es decir, saber qué nos corresponde ser, cómo y dónde nos corresponde estar. En síntesis, se trata de saber nuestro lugar y el sentido que dimana de él, esto es necesario para decidir qué lugar debe ocupar la ciencia en la vida humana.

PALABRAS CLAVES: ciencia-conocimiento, filosofía intercultural, sabiduría.

\begin{abstract}
While producing an intercultural critique to modern science, the author proposes to reflect about science as a meditative thought that recovers its sense. Also, he looks for to where should be the point from which he is able to understand its meaning and role in view of science, taking consciousness about going beyond critique. Therefore, it is not enough to censor or show the deviations of applying science but to exceed the horizon and placing science in its place. However, to know which the place that science belongs is, implies recognizing the meaning of human knowledge and the cognitive activity within the general development of life. In other words, to know what we are supposed to have in our being, and how and when we are expected to be. In sum, this endeavor is about knowing our place and which sense would spring from that point, which is necessary to decide the place that science has in human life.
\end{abstract}

KEYWORDS: science-knowledge, intercultural philosophy, wisdom.

1 Filosofo cubano, Director del Departamento de América Latina de Missio. Ha sido coordinador de los diálogos filosóficos NorteSur y es el principal representante de la filosofía intercultural. 


\section{¿Por qué una meditación?}

En el programa de este simposio que se dedica al tema de "Ciencia y Religión para un mundo sostenible", se justifica su razón de ser con la siguiente argumentación: "Este tercer simposio quiere ser un espacio de reflexión sobre la contribución de la ciencia contemporánea y las nuevas formas de religiosidad en la construcción de un mundo sostenible. Ni una ni otra puede mantenerse al margen de los desastres ecológicos ni del enorme sufrimiento humano, sino que han de implicarse en un compromiso de participación en la paz y en la Tierra.

Hay que restituir los vínculos entre naturaleza, ciencia y religión para generar una sociedad sostenible y superar la separación establecida entre cuerpo y espíritu. Volver a conectar materia y conciencia es la tarea de una cultura emergente que se manifiesta sobre todo en las nuevas formas de ciencia y de religiosidad. Este simposio quiere ahondar en este diálogo ${ }^{1}$.

Pero ¿qué pensar de esta tarea?, ¿cómo reconocerla en su urgencia y nobleza?, ¿estamos preparados para hacer lo que nos pide?

Es verdad que existe la cultura emergente de la que se nos habla en la justificación del simposio, como es verdad también que esa cultura emergente debe ser vista como una manifestación más del empeño constante de la humanidad por buscar caminos alternativos frente a los "señoríos" de turno que amenazan con aplastar sus posibilidades de ser (sobre todo, evidentemente, cuando estas son lo que deben ser, a saber, posibilidades de recreación), y que es verdad, por consiguiente, que disponemos todavía de una cierta memoria que nos auxilia en la percepción de la urgencia y nobleza de esta tarea porque engrandece nuestra sensibilidad humana y contrarresta así el proceso de empequeñecimiento y de rebajamiento de la vida que aceleró la modernidad europea dominante. Sin negar, pues, esta dimensión de la historia, que es en definitiva la que hace razonable la esperanza en nosotros mismos, insistimos, sin embargo, en el sentido de las tres preguntas que acabamos de formular.
No son un recurso retórico. Son indicadores de una triple indigencia que, a nuestro modo de ver, hay que procurar remediar, si es que realmente queremos estar a la altura de esta tarea y de asumirla como un compromiso en el que el "todo" que está en juego es todavía inmensamente mayor que todo lo que nosotros sabemos nombrar como "nuestro" todo.

Es, en primer lugar, la indigencia de un pensar que se queda perplejo ante esta tarea, es decir, que no sabe qué pensar ni qué decir de la misma porque ante esta tarea queda aturdido. Pero ¿no será la indigencia que se muestra en este aturdimiento del pensar señal de que el pensar mismo ha perdido el sentido de su tarea? Pues ¿cómo es posible que el pensar no sepa qué pensar ante una tarea que le pide búsqueda de sentido? Esto es posible solo si el pensar ha olvidado qué significa pensar. Esta es nuestra hipótesis; y, como veremos más adelante, la indigencia del pensar a la que nos referimos es resultado de un proceso histórico que ha llevado a que el pensar no "piense". Remediar esta indigencia requiere, pues, intentar responder a la pregunta de cómo hacer para que nuestro pensar "recobre el conocimiento", el sentido 2 .

En segundo lugar, se trata de una indigencia ontológico-existencial que paradójicamente proviene de las comodidades con que nos acomoda en el mundo la llamada civilización científica o técnica. Pues con ello reducimos nuestras relaciones todas a un nivel de instrumentalidad funcional que crea el espejismo de que todo es asunto de mejoramiento de las comodidades, es decir, de perfeccionar el funcionamiento de la máquina; y cerramos así el horizonte a cuya luz se puede reconocer la necesidad urgente de restituir los vínculos originales, aunque sea al precio del acomodamiento en las funciones del sistema.

$Y$ en tercer lugar es una indigencia ético-espiritual que, como consecuencia de la anterior, nos impide responder con el comportamiento adecuado. Nuestro acomodado ser no está ni preparado ni dispuesto para lo que pide esta tarea de nosotros. A más tardar desde Platón se sabe en Occidente que el comportamiento, la acción humana, es una expresión de la manera en que el ser humano es y se comprende ${ }^{3}$, y si este principio, que

1 Ver programa del “III Simposio: Ciencia y Religión para un mundo sostenible. Una relación recíproca con la naturaleza” de la

"Fundación Filosofia de la Terra i de les Cultures". El presente texto es la ponencia dada en dicho simposio que se celebra del 15

al 16 de diciembre de 2007 en Palma de Mallorca.

2 Cf. Martín Heidegger, ¿Qué significa pensar?, Buenos Aires 1964.

3 Cf. Platón, Cármides, en Obras Completas, Madrid 1960, págs. 274 y sgs. 
en la tradición escolástica se condensó en el famoso lema de "Operari sequitur esse", es cierto, ¿cómo esperar que seres humanos que son y se comprenden desde los parámetros de la civilización científico-técnica puedan actuar de otra manera?

Por esta situación de indigencia en la que está acomodada nuestra condición humana hoy y que, como veremos, tiene mucho que ver con el hecho de que ese acomodamiento es precisamente de base científico-técnica, creemos que la tarea a la que se nos quiere animar con este simposio, tiene que comenzar por un acto de recapacitación del pensar; un acto por el que el pensar se detenga en su actividad de reflexionar sobre cosas para recogerse y pensar qué le significa pensar. A este acto le llamamos meditación; y creemos que es necesario empezar por él, porque, como decíamos, el pensar ya no piensa. Está atrapado en un engranaje de producción instrumental de saberes que en nombre del desarrollo y del progreso lo obliga a entenderse en términos de una función del sistema epistemológico sancionado por la civilización científicotécnica. Y para "recobrar el sentido" necesita precisamente meditar.

Para el tema que nos ocupa se desprende de lo anterior que proponemos pensar la ciencia con un pensar meditativo que recobra el sentido $y$, percatándose del significado de su tarea ante la ciencia, toma conciencia de que debe ir más allá de la crítica ${ }^{4}$. Pues no basta con censurar o poner de manifiesto desviaciones en el uso de la ciencia, sino que hay que sobrepasar este horizonte poniendo a la ciencia en su lugar.

Saber, sin embargo, qué lugar le corresponde a la ciencia, implica saber qué sentido tienen el conocimiento humano y la actividad cognitiva del ser humano en general dentro del desarrollo de la vida, es decir, saber qué nos corresponde ser, cómo y dónde nos corresponde estar. Saber nuestro lugar y el sentido en él, es, pues, necesario para decidir qué lugar debe ocupar la ciencia en la vida humana. Pero el pensar "aturdido" por el impresionante progreso de la ciencia necesita meditar para poder saber este otro saber sobre el sentido de su tarea.

He aquí un resumen de las ideas de fondo en razón de las cuales proponemos no simplemente un pensar la ciencia, sino justamente hacer de ella un objeto de meditación del pensar.

\section{¿De qué ciencia hablamos?}

Por lo que llevamos dicho se comprende que nos estamos refiriendo a la llamada ciencia moderna. Con todo nos parece conveniente aclarar que con este título, a pesar de todas la continuidades que se quieran establecer para salvar un cierto hilo conductor o "una lógica científica" en la historia de la ciencia, entendemos un fenómeno cultural específico que poco tiene que ver con la "episteme" de la antigüedad griega clásica ni con la doctrina - "scientia" de la Edad Media cristiana que entienden la ciencia como un saber de y desde principios fundamentales evidentes por sí mismos y cuyo conocimiento asegura a dicho saber la correspondencia con lo que es verdadero e inmutable, necesario y universal ${ }^{5}$. Y por ser trasmisora de un saber semejante se considera además en esta concepción clásica que la ciencia es un elemento indispensable para la verdadera formación del ser humano, como se ve todavía en los representantes del idealismo alemán y en Wilhelm von Humboldt ${ }^{6}$.

Pero, como decíamos, la ciencia moderna rompe radicalmente con esa concepción al abandonar, debido al giro epistemológico del nominalismo, el principio central de la ciencia clásica que dice "scientia non est singularium", aplicándose precisamente al estudio de fenómenos concretos, experimentables y medibles. Este es el giro que se suele unir con razón a los nombres de Cópernico (1473-1543), Galileo (1571-1642) y Kepler (1571-1630) para marcar el inicio de una nueva idea de la ciencia, es

4 La bibliografía sobre este punto, incluyendo la crítica a la racionalidad científica como método y horizonte de conocimiento, es inmensa y, en parte, también muy conocida. Por eso nos limitamos a mencionar unos pocos títulos que nos parecen especialmente significativos: Karl J aspers, Origen y meta de la historia, Madrid 1965; Kurt Hübner, Kritik der wissenschaftlichen Vernunft, Freiburg 1978; Martin Heidegger, "Die Frage nach der Technik", en Vorträge und Aufsätze, Pfullingen 1954; J osé Ortega y Gasset, Meditación de la técnica, en Obras completas, tomo V, Madrid 1983; J acques Ellul, Le Systèm technicien, Paris 1977; Carl Friedrich von Weizäcker, Der Garten des Menschlichen, München 1977; y Émile Bréhier, Ciencia y humanismo, Murcia 1958.

5 Como autores paradigmáticos recordemos aquí a Aristóteles (Cf. por ejemplo su explicación en Ética a Nicómaco, libro VI) y a Santo Tomás (Cf. por ejemplo su Summa Theologica, q. 1 a. 1-2).

6 Desde Kant a Krause se ve esta concepción en las obras fundamentales de los representantes del idealismo alemán, que son en ese sentido obras en las que la ciencia se comprende como un capítulo en el camino de la "perfectibilidad" del espíritu humano. Sobre Humboldt ver: Eduard Spranger, Wilhelm von Humboldt und die Humanitätsidee, Berlín 1928. 
decir, el inicio de la ciencia (moderna) que sustituye la inteligencia de los principios por la investigación basada en la observación y la experiencia de sectores determinados de la realidad.

Pero acaso lo decisivo para la configuración de la "nueva ciencia" no sea esta opción por el método experimental y analítico, sino el hacer de esta opción el instrumento por excelencia para posibilitar, consolidar y extender el dominio del ser humano sobre la naturaleza. Pues es así como en el marco del nacimiento de la modernidad y de la formación económica del capitalismo la ciencia (moderna) puede sustituir la idea del orden cósmico por una concepción mecánica del mundo en la que se refleja justamente la voluntad de señorío humano sobre la naturaleza. Desde este momento la ciencia será un factor sistémico en la empresa del apoderamiento y del control del mundo por parte de una humanidad cuya autoconciencia de señorío es incompatible con la "tolerancia" del misterio y de lo sagrado en el mundo. $Y$ es por eso que la autoafirmación de esta conciencia de señorío es proporcional con la positivización de la ciencia. El señorío humano no necesita una ciencia reflexiva, sino, por el contrario, una ciencia positivizada que cierre los espacios abiertos de reflexión con el "saber hacer" del técnico.

Pero no es necesario seguir abundando en esta caracterización, pues es un desarrollo conocido y documentado desde muy diversas perspectivas, como muestras, entre otros, los análisis de Edmund Husserl, Herbert Marcuse, J ürgen Habermas o Raimon Panikkar ${ }^{7}$.

Importante es, sin embargo, complementar lo dicho con el dato de que esa positivización de la ciencia mucho tiene que ver con un rasgo que es distintivo en gran medida de la ciencia moderna, a saber, que es una ciencia cuya producción de "saber hacer" la convierte en una fuerza productiva de primer rango. Lo que, a su vez, es el horizonte a cuya luz hay que explicar su significado tan decisivo para todas nuestras "comodidades". Esta ciencia, como señaló, Mario Bunge hace ya mucho tiempo, se ha convertido "en el núcleo de la cultura moderna" 8 .

Si este diagnóstico es correcto, y todo parece indicar que lo es, la ciencia de la que hablamos no es, pues, una manifestación marginal de nuestra cultura. Es verdad que es un fenómeno cultural, generado históricamente, pero con la peculiaridad de haberse convertido en el corazón mismo de la cultura en que crece. La ciencia es definitiva para la cultura mundial en que hoy se mueve el ser humano porque ha decidido el carácter de esa cultura y con ello las pautas normativas en que nos orientamos como sociedad mundial.

Sobre el trasfondo de estas breves aclaraciones, cuya intención ha sido únicamente la de recordar la centralidad peculiar de la ciencia moderna para nosotros hoy, tratemos ahora de ver qué nos puede significar meditar sobre ella.

\section{Para una meditación de la ciencia}

Son conocidos los análisis de Max Weber sobre las consecuencias "modernizantes" que ha tenido la planetarización de la racionalidad científico-técnica occidental para la organización de la vida social y política de la humanidad en los últimos siglos ${ }^{9}$. Y conocidos son también los análisis que, siguiendo en gran parte el horizonte explicativo abierto por Weber, hace J ürgen Habermas con la intención de ilustrar que este proceso de racionalización científico-tecnológica que es nuclear en las sociedades industrializadas, genera un profundo desacoplamiento entre los sistemas de las funciones sociales y el mundo real de la vida del diario, que conduce en el fondo a la colonización de este último ${ }^{10}$.

Estos análisis, como tantos otros que van en esa línea ${ }^{11}$, son ciertamente discutibles en muchos de sus aspectos; pero lo que sí nos parece seguro en ellos, y por eso tomamos aquí su perspectiva de fondo para enmarcar nuestra propuesta de meditación de la ciencia, es que nos ayudan a comprender uno de los problemas fundamentales que plantea hoy la ciencia a la humanidad. Nos referimos al poder

7 Cf. Edmundo Husserl, Die Krise der europäischen Wissenschaften und die transzendentale Phänomenologie, La Haya 1962; Herbert Marcuse, El hombre unidimensional, México 1968; J ürgen Habermas, Technik und Wissenschaft als Ideologie, Frankfurt a./ M 1976; y Raimon Panikkar, L'émancipación de la tecnologie, en Interculture 85 (1984) 22-37, Paz y desarme cultural, Santander 1993, y La dialéctica de la razón armada, en Concordia 9 (1986).

8 Mario Bunge, La ciencia, su método y su filosofía, Buenos Aires 1966, pág. 126.

9 Cf. Max Weber, Wirtschaft und Gesellschaft, Köln 1964, y Gesammelte Aufsätze zur Religionssoziologie I, Tübingen 1988.

10 Cf. J ürgen Habermas, Theorie des kommunikativen Handelns, dos tomos, Frankfurt a./ M 1988

11 Ver por ejemplo, Helmut Schelsky, Der Mensch in der technischen Zivilisation, Hamburg 1961; y Arnold Gehlen, Die Seele im technischen Zeitalter. Sozialpsychologische Probleme in der industriellen Gesellschaft, Frankfurt a. / M 2007. 
y a la autoridad que tiene hoy la ciencia; poder y autoridad que se consideran tan "naturales" que apenas si se cuestiona la legitimidad con que impone sus criterios en los más diversos ámbitos de la sociedad e invade los mundos de la vida.

Tomemos, pues, esta situación de colonización como punto de partida para nuestra meditación. Pero observemos que esto nos obliga a pensar primero, como se desprende de lo ya dicho sobre las tres preguntas formuladas al principio, en qué situación nos encontramos nosotros mismos para pensar la ciencia. Pues debido a la fuerza del monopolio cognitivo que detenta hoy la ciencia como fuente exclusiva de conocimientos válidos y reconocidos como tales, hay buenas razones para suponer que la ciencia no solo coloniza nuestros mundos de vida social y cotidiana, sino también la relación con nosotros mismos $y$, con ello, nuestros "mundos interiores", si se permite llamarles así. Preguntemos entonces si estamos todavía en el nivel de lo que llamamos antes el "pensar aturdido", que sería sin duda una señal clara de que la colonización de la ciencia también ha llegado hasta nuestra propia manera de pensar.

Empezar la meditación por esta pregunta es un recurso metódico para discernir el lugar cognitivo donde realmente está nuestro pensar, es decir, discernir la situación en que pensativamente estamos cuando decimos qué pensamos, para saber en este caso concreto en qué condiciones estamos para pensar la ciencia. Debemos empezar, pues, por tratar de determinar en qué medida la ciencia informa las condiciones desde las que pensamos, entendiendo evidentemente que esa influencia se da también de manera negativa o indirecta, como mostrarían, por ejemplo, los complejos que se tienen frente a la ciencia en las Ilamadas "Humanidades" o las consecuentes luchas por el reconocimiento "científico".

Meditar la ciencia requiere, por tanto, recuperar no solamente la capacidad crítica, sino también la libertad del pensamiento frente a la ciencia, pues solo así se podría pensar la ciencia desde fuera del paradigma cognitivo que ha impuesto. $Y$ de ello precisamente se trata, porque: "¿cómo se podría poner la ciencia en su lugar si seguimos pensándola según los criterios de su paradigma?

Pero debemos aclarar que la recuperación de la libertad del pensamiento frente a la ciencia no quiere decir desentenderse de ella ni negarla, como tampoco significa querer afirmar de otra manera la independencia del pensamiento humano. Quiere decir más bien recuperación de los vínculos fundantes con la naturaleza, con el otro, con lo sagrado, lo divino, etc., que el reduccionismo de la ciencia positivizada ha expulsado de las zonas de experiencia del pensar. Por esta libertad el pensamiento no gana "independencia", sino vinculación, arraigo en un orden que la ciencia redujo a "estructuras" y "funciones". Mas por eso mismo, por esta recuperación de los vínculos, puede pensar la ciencia de otra manera, esto es, ocuparse de su significado en un orden que ya no es simple reproducción de las leyes de la investigación científica, sino que, por el contrario, se revela como el orden que ordena (en el doble sentido de mandar y poner en concierto) el conocimiento humano.

Así, desde esta libertad o apertura de horizontes que da la recuperación de los vínculos fundantes del ser humano, puede el pensar meditar la ciencia con intención, como decíamos, de ir más allá de la crítica de sus reduccionismos y de sus consecuencias sociales y ecológicas, para reordenarla desde sus principios. O sea que una meditación de la ciencia, sin menospreciar las consecuencias que produce la maquinaria científica de una sociedad científico-tecnológica, se concentra en los principios con que la ciencia ha querido suplantar o compensar la pérdida de seguridad y de serenidad que significó en Europa el tránsito del orden cósmico clásico al "constructivismo" moderno. En resumen, se trataría de meditar sobre el sentido de estos principios:

- Principio epistemológico: priorizar -supuesta la dualidad sujeto-objeto de conocimiento- el método del logos "investigador" para el que la búsqueda del sentido se disuelve en la explicación de las leyes a que obedece la "construcción arquitectónica de la realidad".

- Principio "cosmológico": puesta en marcha de una visión "mecánica" de la naturaleza. Es decir, desnaturalización de la ciencia.

- Principio antropológico: afirmación -como consecuencia de los dos anteriores- de un humanismo antropocéntrico que redefine el puesto del ser humano en el cosmos y que prioriza el apoderamiento como línea de humanización. 
Revista CUHSO volumen $14 \mathrm{n} \div 1$

- Principio de independencia cultural: el saber científico no está determinado por factores sociales ni culturales y se desarrolla por eso como un conocimiento objetivo de validez universal. De donde se sigue:

- Principio de la despersonalización: el conocimiento científico se produce con independencia del trasfondo biográfico (deseos, intereses, sueños, miedos, etc. ), de las personas implicadas en el proceso de investigación (que es un proceso institucionalizado e independiente de la corporalidad humana). Por eso se podría hablar aquí también de principio de la descorporalización ${ }^{12}$ de la ciencia, que sería la otra cara de la desnaturalización.

- Principio del progresismo: aplicación metódica y sistemática de la investigación en el sentido de una lucha abierta contra todo límite.

La meditación sobre el sentido de estos principios, pero también sobre las consecuencias sociales, ecológicas, antropológicas y religiosas que ha tenido el desarrollo de una ciencia afincada en principios semejantes, nos llevaría a comprender, a nuestro modo de ver, que la reconstrucción de los vínculos entre naturaleza, ciencia y religión a la que quiere contribuir este simposio, es imposible si no se cambian los principios de la ciencia moderna en su aparato dominante, pues son precisamente la expansión sistemática y la aplicación rigurosa de esos principios los que han roto el ordenamiento natural y sus vínculos de reciprocidad.

Mas ¿puede la ciencia cambiar sus principios? Sí puede, aunque el precio de ese cambio esel de una refundamentación holística, intercultural e interreligiosa de la ciencia. Aquí es importante, es más, indispensable el diálogo abierto entre las diferentes culturas del saber de la humanidad, pues la refundamentación requerida supone abrirse a otros principios y a otros fines para recuperar la pluralidad de fundamentos que "reubica" el edificio de la ciencia moderna.

Refundar la ciencia es, pues, el desafío de recolocarla en el tejido de los vínculos de reciprocidad que nos recuerdan las memorias culturales y religiosas de la humanidad. Es, como decíamos, "ponerla en su lugar" para que deje de ser la fuerza que finaliza toda capacidad cognitiva humana. Es, dicho de otra manera, replantear el problema del ideal del conocimiento científico.

Este problema nos es, sin embargo, una cuestión solo epistemológica. Acaso es ante todo una cuestión ético-política. Pues todo parece indicar que la ciencia que hace y que financia una sociedad (o determinados grupos de poder) depende en gran medida de la forma en que se haya respondido a la pregunta de qué queremos ser y de cómo queremos estar en el mundo.

Por esta razón, y lógicamente por las alteraciones que ha provocado la ciencia moderna en nuestra relación con nosotros mismos, una meditación (filosófica) sobre la ciencia no debería escatimar la consideración de la cuestión sobre si la forma en que somos es una forma que, por aceptar su conformación por los vínculos fundantes de la existencia viviente, vive en conformidad y en paz con el ordenamiento de los vínculos; o si, por el contrario, es una forma en continua insatisfacción que nunca se conforma y hace de su vida un obstinado proceso de "consumir" la vida.

Responder a esta pregunta nos parece decisivo para nuestras posibilidades de refundar la ciencia y de reordenarla de acuerdo con fines nuevos. 


\section{UNIVERSIDAD CATÓLICA DE TEMUCO}

\section{BIBLIOGRAFÍA}

ARISTÓTELES (Cf. Por ejemplo su explicación en Ética a Nicómaco, libro VI) y a su Santo Tomás (Cf. Por ejemplo su Summa Theologica, q. 1 a. 1-2)

BRÉHIER, É. (1958), Ciencia y humanismo, Murcia.

BUNGE, M. (1966), La ciencia, su método y su filosofía. Buenos Aires, pág. 126.

ELLUL, J. (1977), Le Systém technicien. Paris.

FIEDRICH VON EWIZACKER, C. (1977) Der Garten des Menschlichen. München.

GEHLEN, A. (2007), Die Seele im technischen Zeitalter. Sozialpsychologische Probleme in der industriellen Gesellschaft. Frankfurt a./ M.

GEHLEN, A. (1965), Technik im technischen Zeitalter. Frankfurt a./ M.

HABERMAS, J . (1976), Technik und Wissenschaft als Ideologie. Frankfurt a./M.

HABERMAS, J. (1988), Theorie des kommunikativen Handelns. Frankfurt a./ M.

HEIDEGGER, M (1954), “Die Frage nach der Rechnik", en Vorträge und Aufsätze, Pfullingen.

HEIDEGGER, M. (1964) ¿Qué significa pensar? Buenos Aires.

HÚBNER, K (1978), Kritik der wissenschaftlichen Vernunft. Freiburg.

HUSSEL, E (1962), Die Krise ser europäischen Wissenschaften und die tranzendentale Phänomenologie, La Haya.

JASPERS, K. (1965) Origen y meta de la historia, Madrid.

MARCUSE, H. (1968), El Hombre unidimensional, México.

ORTEGA Y GASSET, J . (1983), Meditación de la técnica, en Obras Completas, tomo V. Madrid.

PANIKKAR, $R$ (1984), "L'émancipation de la tecnologie", en Interculture 85 (1984) 22-37, Paz y desarme cultural, Santander 1993 y “La dialéctica de la razón armada", en Concordia 9 (1986).

PLATÓN (1960), Cármides, en Obras Completas, Madrid, págs. 274 y sgs.

ACHELSKY, H. (1961), Der mensch in der technischen Zivilisation, Hamburg.

SPRANGER, E. (1928), Wilhelm von Humboldt und die Humanitätsidee, Berlín.

WEBER, M (1988), Wirtschaft und Gesell sschaft, Köln 1964 y Gesammelte Aufsätze zur Religionssoziologie I, Tübingen. 